\title{
Stability of Communications Networks in the Presence of Delays
}

\author{
Chaouki T. Abdallah*and John Chiasson \\ ECE Department \\ 1508 Middle Drive \\ 414 Ferris Hall \\ University of Tennessee \\ chiasson@utk.edu, chaouki@utk.edu \\ Marco Ariola \\ Dipartimento di Informatica e Sistemistica \\ Università degli Studi di Napoli Federico II \\ Napoli, Italy \\ ariola@unina.it
}

May 15, 2001

\begin{abstract}
In this paper, we present a study of the destabilizing effects of delay in communications networks. We the apply results from a companion paper to find the range of delays for which a Smith predictor controller guarantees the stability of an ATM/ABR network.
\end{abstract}

\section{Introduction}

Congestion control in the Available Bit Rate (ABR) class of Asynchronous Transfer Mode (ATM) networks poses interesting challenges due to the presence of multiple-delays, magnitude and rate constraints on the inputs, amplitude limitation on the state, and additive disturbances. The ABR class is designed as a best-effort class for applications such as file transfer and email. Thus, no service guarantees are required, but the source of data packets controls its data rate, using a feedback signal provided by switches downstream that measure the congestion of the network. Due to the presence of this feedback, many classical and advanced control theory concepts have been suggested to deal with the congestion control problem in the ATM/ABR case.

${ }^{*}$ The research of C.T. Abdallah is partially supported by NSF INT-9818312 
We will focus our modeling on one particular queue in the network which is associated with a link shared by many virtual circuits [10]. A virtual circuit is established for any two stations wishing to communicate, by informing all switches between them of their requirements. Assuming that the flow of packets is conserved, the queue level model for each buffer in the ATM network (as proposed in [10]) is

$$
\dot{x}(t)=\sum_{i=1}^{n} u_{i}\left(t-T_{i}\right)-d(t)
$$

where

- $x(t)$ is the queue level associated with the considered link;

- $n$ is the number of virtual circuits sharing the queue associated with the considered link;

- $u_{i}(t)$ is the inflow cell rate caused by the $i$ th virtual circuit;

- $T_{i}$ is the propagation delay from the $i$ th source to the queue, and is usually uncertain;

- $d(t)$ is the rate of packets leaving the queue.

More details on this model and its physical interpretation is available from [7]. Equation (1) appears to be linear since the saturation effect due to the limited buffer capacity has been neglected. Our control design however, must ensure that this condition is actually satisfied, so that the controller is not saturated. The author in [7] proposes the Smith predictor control scheme of Figure 1. The objectives of the control law proposed in [7] are to guarantee

- Stability ${ }^{1}$ :

$$
x(t) \leq r^{0} \quad t \geq 0
$$

where $r^{0}$ is the queue capacity. This condition guarantees no cell loss and is not the usual stability requirement;

- Full Link Utilization:

$$
x(t)>0 \quad t>T_{t r}
$$

The time $T_{t r}$ in (2) mainly accounts for the transient time of the dynamics. Let $\hat{T}_{i}$ denote the estimated values of the delays $T_{i}$. Assuming that $\hat{T}_{i}=T_{i}$ and disregarding for the moment the presence of disturbance $d(t)$, the scheme in Figure 1 can be shown to be equivalent to the one in Figure 2[7]. In this case, the designer knows exactly the value of all delays $T_{i} ; i=1, \cdots, n$, and the closed-loop system exhibits the following nice properties.

\footnotetext{
${ }^{1}$ The network community refers to this as a "stability" condition. Stability in the dynamic systems sense will guarantee BIBO stability and then care must be taken to ensure the bound $r^{0}$ is not violated.
} 




Figure 1: The controlled system.

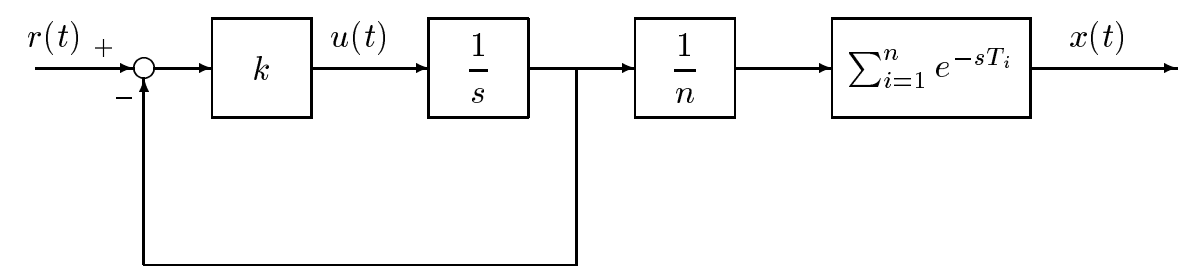

Figure 2: The desired closed-loop system.

- With $r(t)=r^{0} \cdot 1(t)(1(t)$ is the unit step) denoting the desired reference level of the queue, and $d(t)=a \cdot 1(t)$, with $0 \leq a \leq 1$ the stability property (boundedness) is satisfied.

- Further, with $d(t)=a \cdot 1(t)$, with $0 \leq a \leq 1$ the full utilization property is satisfied, provided that [7]

$$
r^{0}>a\left(\frac{1}{k}+\frac{1}{n} \sum_{i=1}^{n} T_{i}\right) .
$$

The technique which is used in order to obtain the desired closed-loop system is the well-known Smith's principle [15][16].

The main drawback of the approach of [7] is that it assumes that the propagation delays $T_{i}$ are exactly known. When this is not the case, even stability, in the sense defined above $^{2}$, can be lost. In order to illustrate this limitation

${ }^{2}$ That is, $x(t) \leq r^{0}$ is violated. 
and the potential of losing stability (boundedness), a simulation was performed. Assuming that $n=4, T_{1}=10 \mathrm{sec}, T_{2}=30 \mathrm{sec}, T_{3}=60 \mathrm{sec}, T_{4}=120$ sec, $k=0.1$, and $r^{0}=40$, the stability condition is satisfied (see Figure 3 ) if $\hat{T}_{i}=T_{i} i=1, \ldots 4$.

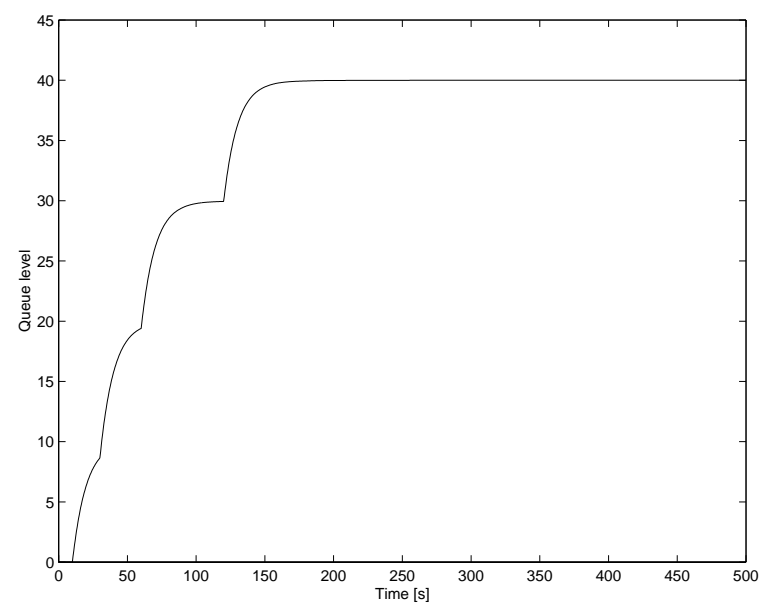

Figure 3: Queue level when the propagation delays are assumed to be known.

Nest, the actual delays $T_{i} ; i=1, \cdots, n$ are perturbed by $5 \%$, without modifying their assumed values $\hat{T}_{i} ; i=1, \cdots, n$. As shown in Figure 4, stability is lost since $x(t)$ is greater than $r^{0}$ during some time intervals. Stability is regained however if the controller gain $k$ is changed to $k=0.01$ ( see Figure 5 ) at the expense of a slower response. When the $T_{i}$ s however are not known, the designer cannot a priori design a Smith-predictor-type controller in order to guarantee the desired degree of stability and performance. Moreover, even BIBO stability may be lost if one does not take the effects of the uncertainty in the delays.

In the following, we use our results from the companion paper [3] to study the stability of the ATM/ABR system when the delays are uncertain.

\section{Robust Stability for the ATM model}

The transfer function of the ATM system is

$$
X(s)=\frac{\frac{k}{n} \sum_{i=1}^{n} e^{-s T_{i}}}{s+k-\frac{k}{n}\left(\sum_{i=1}^{n} e^{-s T_{i}}-e^{-s \hat{T}_{i}}\right)} R(s)-\frac{s+\frac{k}{n}\left(n-\sum_{i=1}^{n} e^{-s \hat{T}_{i}}\right)}{s\left(s+k-\frac{k}{n}\left(\sum_{i=1}^{n} e^{-s T_{i}}-e^{-s \hat{T}_{i}}\right)\right)} D(s)
$$

First note that the transfer function

$$
\frac{s+\frac{k}{n}\left(n-\sum_{i=1}^{n} e^{-s \hat{T}_{i}}\right)}{s}
$$




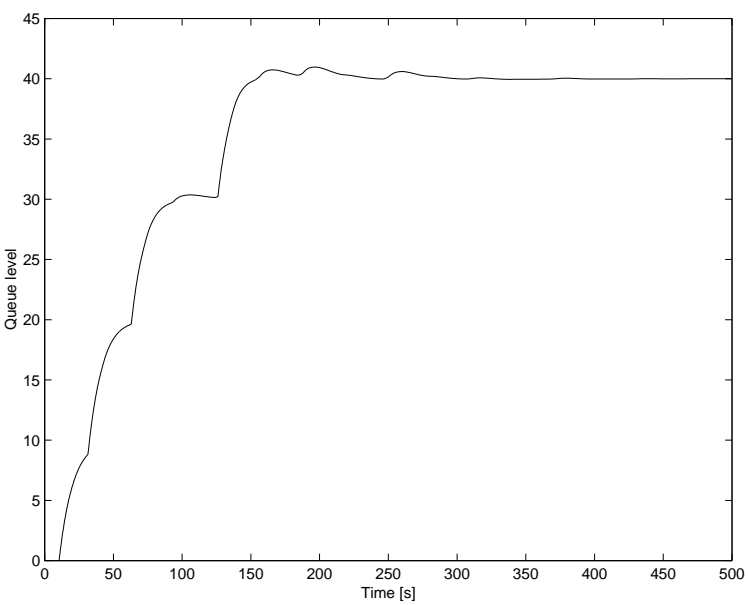

Figure 4: Queue level when the propagation delays are perturbed by $5 \%$.

is stable since the only possible pole location is $s=0$ and there it follows that

$$
\begin{aligned}
\left.\frac{s+\frac{k}{n}\left(n-\sum_{i=1}^{n} e^{-s \hat{T}_{i}}\right)}{s}\right|_{s=0} & =\lim _{s \rightarrow 0} \frac{s+\frac{k}{n}\left(n-\sum_{i=1}^{n} e^{-s \hat{T}_{i}}\right)}{s} \\
& =\lim _{s \rightarrow 0} \frac{s+\frac{k}{n}\left(n \sum_{i=1}^{n} \hat{T}_{i}\right) s}{s} \\
& =1+k \sum_{i=1}^{n} \hat{T}_{i} \neq \infty
\end{aligned}
$$

Consequently, the stability of the ATM system is reduced to studying the stability of $s+k-\frac{k}{n}\left(\sum_{i=1}^{n} e^{-s T_{i}}-e^{-s \hat{T}_{i}}\right)$. In this section, being stable means

$$
s+k-\frac{k}{n}\left(\sum_{i=1}^{n} e^{-s T_{i}}-e^{-s \hat{T}_{i}}\right) \neq 0 \text { for } \operatorname{Re}(s) \geq 0
$$

In order to analyze the stability of this system, some further refinements in the model are now made. Let $T_{i}=m_{i} h_{0}, \hat{T}_{i}=m_{i} h$ for $i=1, \ldots, n$ where the $m_{i}$ are positive integers ${ }^{3}$. Define $c(z) \triangleq \sum_{i=1}^{n} z^{m_{i}}$ so that $c\left(e^{-s h_{0}}\right)=$ $\sum_{i=1}^{n} e^{-s T_{i}}, c\left(e^{-s h}\right)=\sum_{i=1}^{n} e^{-s \hat{T}_{i}}$. The characteristic equation of the ATM system (3) may be rewritten as

$$
a\left(s, e^{-h_{0} s}, e^{-h s}\right) \triangleq a_{0}(s)+a_{1}(s)\left[c\left(e^{-s h_{0}}\right)-c\left(e^{-s h}\right)\right]
$$

where $a_{0}(s)=s+k, a_{1}(s)=-k / n$. Here the delays $h_{0}, h$ are non-commensurate in general. By design, the compensator $G_{c}(s)=k$ is chosen so that (4) is stable

\footnotetext{
${ }^{3}$ For all practical purposes, there will always be $h_{0}, h$ such that the delays can be written as integer multiples of these values.
} 


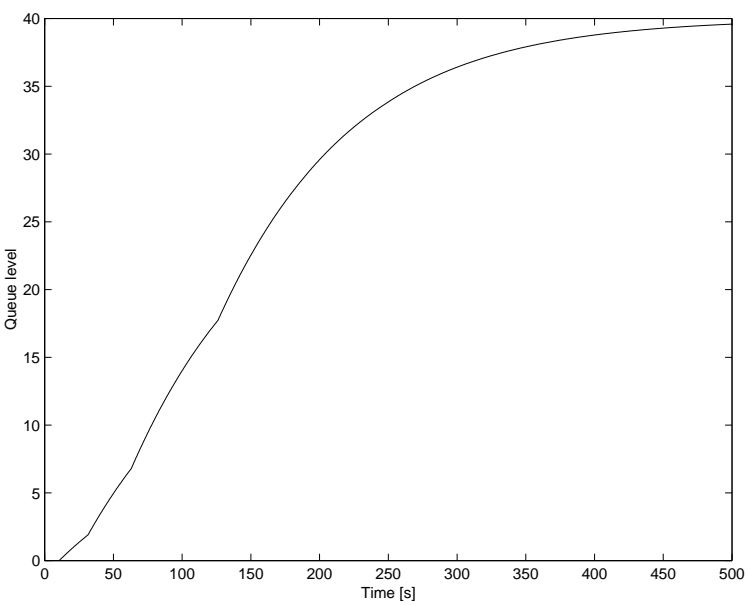

Figure 5: Queue level when the propagation delays are perturbed by $5 \%$ and $k=0.01$.

for $h=h_{0}$, that is, $a_{0}(s)$ is stable. The interest here is to determine the range of values of $h$ about $h_{0}$ for which (4) remains stable. As $h$ is decreased (or decreased) in value from $h_{0}$, there is a first value $h^{*}$ for which the roots of (4) are on the $j \omega$ axis, that is,

$$
a\left(s, e^{-h_{0} s}, e^{-h^{*} s}\right) \triangleq a_{0}(s)+a_{1}(s)\left(c\left(e^{-s h_{0}}\right)-c\left(e^{-s h^{*}}\right)\right)=0
$$

for some $s^{*}=j \omega^{*}$.

To exploit this idea $\left(z_{0}=e^{-s h_{0}}, z=e^{-s h}\right)$, define $\left(m=\max _{i}\left\{m_{i}\right\}\right)$

$$
\begin{aligned}
a\left(s, z_{0}, z\right) & =a_{0}(s)+a_{1}(s)\left(c\left(z_{0}\right)-c(z)\right), c(z)=\sum_{i=1}^{n} z^{m_{i}} \\
\tilde{a}\left(s, z_{0}, z\right) & \triangleq z_{0}^{m} z^{m} a\left(-s, 1 / z_{0}, 1 / z\right)=z_{0}^{m} z^{m}\left[a_{0}(-s)+a_{1}(-s)\left(c\left(1 / z_{0}\right)-c(1 / z)\right)\right] \\
& =z_{0}^{m} z^{m} a_{0}(-s)+a_{1}(-s)\left(z^{m} c\left(z_{0}\right)-z_{0}^{m} c(z)\right)
\end{aligned}
$$

Solving $a\left(s, z_{0}, z\right)=0$ to get $c(z)=\left(a_{0}(s)+a_{1}(s) c\left(z_{0}\right)\right) / a_{1}(s)$ and substituting this into $\tilde{a}\left(s, z_{0}, z\right)=0$ gives

$$
\begin{aligned}
0 & =z_{0}^{m} z^{m} a_{0}(-s)+a_{1}(-s)\left(z^{m} c\left(z_{0}\right)-z_{0}^{m} \frac{a_{0}(s)+a_{1}(s) c\left(z_{0}\right)}{a_{1}(s)}\right) \\
z^{m} & =\frac{z_{0}^{m}\left(a_{1}(-s) a_{0}(s)+a_{1}(s) a_{1}(-s) c\left(z_{0}\right)\right)}{\left(z_{0}^{m} a_{1}(s) a_{0}(-s)+a_{1}(-s) a_{1}(s) c\left(z_{0}\right)\right)} \\
& =\frac{a_{1}(-s) a_{0}(s)+a_{1}(s) a_{1}(-s) c\left(z_{0}\right)}{a_{1}(s) a_{0}(-s)+a_{1}(-s) a_{1}(s) c\left(1 / z_{0}\right)}, \quad z_{0}^{-m} c\left(z_{0}\right) \equiv c\left(1 / z_{0}\right)
\end{aligned}
$$

With $s=j \omega, z=e^{-j h \omega}, z_{0}=e^{-j h_{0} \omega}$ this becomes 


$$
\begin{aligned}
z^{m} & =e^{-j m h \omega}=\frac{a_{1}(-j \omega) a_{0}(j \omega)+a_{1}(j \omega) a_{1}(-j \omega) c\left(e^{-j h_{0} \omega}\right)}{a_{1}(j \omega) a_{0}(-j \omega)+a_{1}(-j \omega) a_{1}(j \omega) c\left(e^{j h_{0} \omega}\right)} \\
& =\left|\frac{a_{1}(-j \omega) a_{0}(j \omega)+a_{1}(j \omega) a_{1}(-j \omega) c\left(e^{-j h_{0} \omega}\right)}{a_{1}(j \omega) a_{0}(-j \omega)+a_{1}(-j \omega) a_{1}(j \omega) c\left(1 / e^{-j h_{0} \omega}\right)}\right| e^{j \angle \frac{a_{1}(-j \omega) a_{0}(j \omega)+a_{1}(j \omega) a_{1}(-j \omega) c\left(e^{-j h_{0} \omega}\right)}{a_{1}(j \omega) a_{0}(-j \omega)+a_{1}(-j \omega) a_{1}(j \omega) c\left(e^{j h h_{0} \omega}\right)}} \\
& =e^{2 j \angle\left(a_{1}(-j \omega) a_{0}(j \omega)+a_{1}(j \omega) a_{1}(-j \omega) c\left(e^{-j h_{0} \omega}\right)\right)}
\end{aligned}
$$

and solving for $z$

$$
z=e^{-j h \omega}=e^{j \frac{2 \angle\left(a_{1}(-j \omega) a_{0}(j \omega)+a_{1}(j \omega) a_{1}(-j \omega) c\left(e^{-j h_{0} \omega}\right)\right)+2 \pi k}{m}}, k=0,1, \ldots, m-1
$$

By definition, for $h=h^{*}$ corresponds to $a\left(s, e^{-h_{0} j \omega}, e^{-h^{*} j \omega}\right)=0$ for some $s^{*}=j \omega^{*}$ where $e^{-h^{*} j \omega}$ is given by the right-hand side of $(6)$ for $k=0,1, \ldots, m-1$. Thus, the right-hand side of (6) is substituted into (5) to get

$$
0=a_{0}(j \omega)+\left.a_{1}(j \omega)\left[c\left(e^{-j h_{0} \omega}\right)-c\left(e^{-j h^{*} \omega}\right)\right]\right|_{e^{-j h^{*} \omega}=\operatorname{rhs} \text { of }(6)}
$$

For each $k=0,1, \ldots, m-1$ this equation is solved for the corresponding $\omega_{k i}$, $i=1,2, \ldots$ which are then substituted back into (6) to get

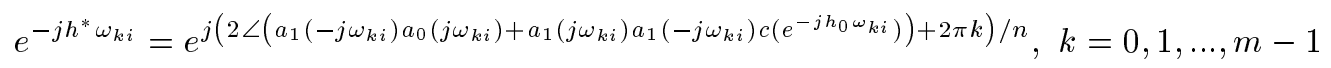

Finally, for each $\omega_{k i}$ this is solved $h_{k i}^{*}$. Then

$$
\begin{aligned}
& h_{1}^{*}=\max _{k, i}\left\{h_{k i}^{*}: h_{k i}^{*}<h_{0}\right\} \\
& h_{2}^{*}=\min _{k, i}\left\{h_{k i}^{*}: h_{k i}^{*}>h_{0}\right\} .
\end{aligned}
$$

Finally, for $h_{1}^{*}<h<h_{2}^{*}$

$$
a\left(s, e^{-h_{0} s}, e^{-h s}\right) \triangleq a_{0}(s)+a_{1}(s)\left[c\left(e^{-s h_{0}}\right)-c\left(e^{-s h}\right)\right] \neq 0 \text { for } \operatorname{Re}\{s\} \geq 0
$$

and

$$
\begin{aligned}
& a\left(s, e^{-h_{0} s}, e^{-h_{1}^{*} s}\right)=0 \text { for some } s=j \omega \\
& a\left(s, e^{-h_{0} s}, e^{-h_{2}^{*} s}\right)=0 \text { for some } s=j \omega
\end{aligned}
$$

Therefore, our approach provides us with a range for the delay within which stability of the closed-loop ATM/ABR system is guaranteed. In the final version of the paper, we will provide numerical example and simulations to illustrate our results. 


\section{Conclusions}

In this paper, we have applied results form our companion paper [3] in order to exactly determine the range of delays for which Smith predictor controllers remain stabilizing. The results are specialized to ATM/ABR networks but can also be applied in many areas of queuing theory and communications.

\section{References}

[1] R. Bellman and K.L. Cooke, Differential-Difference Equations, New York: Academic, 1963.

[2] J.N. Chiasson, S.D.Brierley, and E.B. Lee, "A simplified derivation of the Zeheb-Walach 2-D stability test with applications to time-delay systems," IEEE Transactions on Automatic Control, April 1985.

[3] J.N. Chiasson, and C.T. Abdallah, "Robust Stability of Time Delay Systems: Theory", Submitted to 3rd IFAC Workshop on Time Delay Systems, Santa Fe, NM, December 8-10, 2001.

[4] D. Hertz, E.I. Jury, and E. Zeheb, "Simplified analytic stability test for systems with commensurate time delays," IEE Proceedings, vol. 131, part D, January 1984.

[5] E.W. Kamen, "Linear systems with commensurate time delays: Stability and stabilization independent of delay," IEEE Transactions on Automatic Control, volume AC-27, pp. 367-375, April 1982.

[6] R. Datko, "A procedure for determination of the exponential stability of certain differential-difference equations," Quarterly Applied Mathematics, October 1978.

[7] S. Mascolo, "Smith's Principle for Congestion Control in High-Speed Data Network", IEEE Trans. Automatic Control, Vol. 45, N0.2, pp. 358-364, 2000.

[8] K.L. Cooke and J.M. Ferreira, "Stability conditions for linear retarded functional differential equations," Journal of Mathematical Analysis and Applications, Volume 96, Number 2, October 1983.

[9] B.J. Petterson, R.D. Robinett, J.C. Werner, "Lag-stabilized force feedback damping," Sandia National Laboratories, SAMD91-0194, UC-406 Albuquerque, NM 1991.

[10] D. Cavendish, S. Mascolo, M. Gerla, "SP-EPRCA: an ATM rate based congestion control scheme based on a Smith predictor", preprint.

[11] J.N. Chiasson, "A method for computing the interval of delay values for which a differential-delay system is stable," IEEE Transactions on Automatic Control, Volume 33, Number 12, December 1988. 
[12] S. I. Niculescu and C. T. Abdallah, "Delay effects on static output feedback stabilization", Proceedings of the IEEE CDC, Sydney, Australia, pp. 53-54.

[13] C.T. Abdallah, P. Dorato, J. Benitez-Read and R. Byrne, "Delayed positive feedback can stabilize oscillatory systems," Proceedings of the American Control Conference, San Francisco CA, pp. 3106-3107, 1993.

[14] B. Ataşlar, P.F. Quet, A. İftar, H. Öbay, T. Kang and S. Kalyanaraman, "Robust rate-based flow controllers for high-speed networks: the care of uncertain time-varying multiple time-delays", preprint.

[15] O.J.M. Smith, "Closed Control of Loops with Dead Time." Chemical Engineering Progress, 53(5), 217-219 (1957)

[16] H.M. Power and R. J. Simpson, Introduction to Dynamics and Control, McGraw Hill, 1978. 